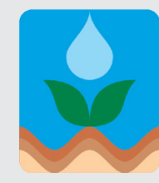

Revista Brasileira de Engemharia Agrícola e An

\title{
Proposta metodológica para projeto de redes hidrométricas: Parte I - Espacialização não tendenciosa dos dados hidrológicos
}

\author{
${ }^{1}$ TSA/IFES, Colatina, ES. E-mail: abrahao@ifes.edu.br (Autor correspondente) \\ ${ }^{2}$ DEA/UFV, Viçosa, MG. E-mail: demetrius@ufv.br \\ ${ }^{3}$ DEA/UFV, Viçosa, MG. E-mail: g.sediyama@ufv.br \\ ${ }^{4}$ DTR/UFRPE, Recife, PE. E-mail: abelardo.montenegro@yahoo.com.br \\ ${ }^{5}$ DEF/UFV, Viçosa, MG. E-mail: cribeiro@ufv.br \\ ${ }^{6}$ CEng/UFPel, Pelotas, RS. E-mail: hugo.guedes@ufpel.edu.br
}

Abrahão A. A. Elesbon ${ }^{1}$, Demetrius D. da Silva ${ }^{2}$, Gilberto C. Sediyama ${ }^{3}$, Abelardo A. A. Montenegro ${ }^{4}$, Carlos A. A. S. Ribeiro ${ }^{5}$ \& Hugo A. S. Guedes ${ }^{6}$

\section{Palavras-chave:}

análise geoestatística

análise hidrológica

monitoramento hidrométrico

\section{R E S U M O}

Neste estudo a aplicação da primeira parte da proposta metodológica para projeto de redes hidrométricas teve, por objetivo, a espacialização não tendenciosa dos dados hidrométricos das redes fluviométrica e pluviométrica existentes na bacia hidrográfica do rio Doce (BHRD) a partir da análise estatística descritiva e da análise geoestatística. Utilizaramse, neste estudo, as variáveis vazão específica mínima média de sete dias consecutivos e período de retorno de 10 anos $\left(\mathrm{q}_{7,10}\right)$ além da precipitação total do semestre mais seco $\left(\mathrm{P}_{\mathrm{ss}}\right)$. Observou-se, aplicando a metodologia desenvolvida que, de forma geral, os valores de $\mathrm{q}_{7,10}$ na BHRD diminuem de oeste para leste com variação de 0,51 a $8,47 \mathrm{~L} \mathrm{~s}^{-1} \mathrm{~km}^{-2}$ enquanto a variável $\mathrm{P}_{\mathrm{ss}}$ apresentou maiores valores na região de cabeceira do rio Piranga e no baixo Doce e valores mínimos na região central da bacia com variação de 147 a $243 \mathrm{~mm}$. A aplicação da metodologia proposta permitiu a espacialização não tendenciosa das variáveis em estudo na BHRD, possibilitando estimar dados em regiões não monitoradas e com menor incerteza.

\section{Key words:}

geostatistical analysis hydrologic analysis hydrometric monitoring

\section{Methodological proposal for hydrometric network design: Part I - Unbiased spatialization of hydrological data}

\begin{abstract}
A B S T R A C T
In this study, the implementation of the first part of the proposed methodology for hydrometric network design aimed to the unbiased spatialization of hydrometric data of fluviometric and rainfall networks existing in the Doce river basin (DRB), from the descriptive statistical analysis and geostatistical analysis. Average minimum specific flow of seven consecutive days and return period of 10 years $-\mathrm{q}_{7,10}$ and total precipitation of half dry - $\mathrm{P}_{\mathrm{ss}}$ variables were used in this study. Applying the developed methodology it was observed that the values of $\mathrm{q}_{7,10}$ in $\mathrm{DRB}$, in general, decrease from west to east, ranging from 0.51 to $8.47 \mathrm{~L} \mathrm{~s}^{-1} \mathrm{~km}^{-2}$. The variable $\mathrm{P}_{\mathrm{ss}}$ showed higher values in the region of the head waters and low course of DRB and minimum values in the central region, ranging from 147 to $243 \mathrm{~mm}$. The proposed methodology allow a unbiased spatialization of study variables in DRB, allowing to estimate data in areas not monitored with lower uncertainty.
\end{abstract}

\section{INTRODUÇÃO}

A Lei $n^{\circ}$ 9.433/1997, que instituiu a Política Nacional de Recursos Hídricos (PNRH) determina, em seu artigo $1^{\circ}$ inciso V, que: "A bacia hidrográfica é a unidade territorial para implementação da PNRH e atuação do Sistema Nacional de Gerenciamento de Recursos Hídricos (SINGREH)". Esta lei preconiza, em seu artigo $2^{\circ}$, inciso II, que a utilização dos recursos hídricos deve ocorrer de forma racional e integrada visando ao desenvolvimento sustentável.

Analisando o estágio atual da PNRH, a Secretaria de Recursos Hídricos e Ambientes Urbanos do Ministério do Meio Ambiente (SRHU/MMA) incentiva o desenvolvimento de ferramentas de suporte à decisão com a participação e a colaboração dos diversos atores sociais envolvidos no processo de gestão de recursos hídricos brasileiros.

A atuação do SINGREH só poderá ser efetuada com sucesso em caso de se dispuser de mecanismos para implementação de uma base de dados hidrológicos confiáveis e de fácil manuseio. A justificativa para o desenvolvimento de ferramentas nesta área se dá sobretudo por se tratar de um assunto de alta relevância ambiental, política e socioeconômica. Eventos hidrológicos extremos, como secas prolongadas e inundações originam, frequentemente, grandes transtornos no território nacional.

Por outro lado, aspectos científicos importantes estão vinculados a uma rede hidrométrica de qualidade: previsão 
climática (Anctil et al., 2006; Coulibaly, 2006; Mishra et al., 2009; Machado \& Rocha, 2011); base de dados hidrológicos para projetos de engenharia (Coulibaly \& Evora, 2007); simulações e calibrações de modelos hidrológicos (Valeriano et al., 2006; Ludwig \& Schneider, 2006; Rogalus \& Ogden, 2007; Bardossy \& Das, 2008; Westcott et al., 2008; Oliveira et al., 2010; Elesbon et al., 2011); redes de alerta em tempo real contra eventos extremos (Gerstner \& Heinemann, 2008); avaliação da qualidade da água (Guedes et al., 2012) entre outros.

Para uma gestão adequada integrada dos recursos hídricos, prevista em lei, faz-se necessário dispor de informações de boa qualidade, seja no tempo quanto no espaço, contemplando diversos setores envolvidos. Tendo em vista esta premissa, a otimização da rede hidrometeorológica de monitoramento hidrométrico existente se torna importante, necessária e urgente sempre que as demandas, tal como os conflitos, aumentam.

A ampliação da rede de monitoramento hidrométrico, prevista pela Resolução ANEEL/ANA nº 003/2010 e as revisões periódicas recomendadas pela Organização Meteorológica Mundial, devem vir acompanhadas de procedimentos técnicos que atendam às demandas nacionais e internacionais.

Neste sentido, este artigo objetivou apresentar a primeira parte da metodologia desenvolvida para o projeto de redes hidrométricas em bacias hidrográficas dando ênfase à espacialização de forma não tendenciosa, a partir da análise estatística descritiva e da análise geoestatística dos dados hidrometeorológicos das redes fluviométrica e pluviométrica existentes na bacia hidrográfica do rio Doce (BHRD).

\section{Material e Métodos}

A BHRD integra a região hidrográfica do Atlântico Sudeste, situando-se entre os paralelos $17^{\circ} 45^{\prime}$ e $21^{\circ} 15^{\prime} \mathrm{S}$ e os meridianos $39^{\circ} 30^{\prime}$ e $43^{\circ} 45^{\prime} \mathrm{W}$, com altitude média de $578 \mathrm{~m}$. A área de drenagem da bacia é de aproximadamente $83.400 \mathrm{~km}^{2}$, dos quais $86 \%$ pertencem ao estado de Minas Gerais e $14 \%$ ao estado do Espírito Santo. A nascente do rio principal se encontra na serra da Mantiqueira, Minas Gerais. O rio Doce percorre cerca de 850 $\mathrm{km}$ até desaguar no Oceano Atlântico, no povoado de Regência, Município de Linhares, Espírito Santo (PIRH, 2013).

\section{Base de dados e aplicativos}

O trabalho foi desenvolvido com base nas séries de dados de 61 estações fluviométricas e 112 estações pluviométricas pertencentes às redes hidrometeorológicas gerenciadas pela Agência Nacional de Águas - ANA e pelo Instituto Nacional de Meteorologia - INMET (Figura 1). As séries empregadas são constituídas por dados anuais consistidos, correspondentes a um período-base de 30 anos (1976 a 2005).

Neste estudo foram consideradas as seguintes variáveis hidrológicas: vazão específica mínima média de sete dias consecutivos e período de retorno de 10 anos $-\mathrm{q}_{7,10}$ e precipitação total do semestre mais seco $-\mathrm{P}_{\mathrm{ss}}$. A variável $\mathrm{q}_{7,10}$ foi escolhida por se tratar da vazão mínima de referência adotada

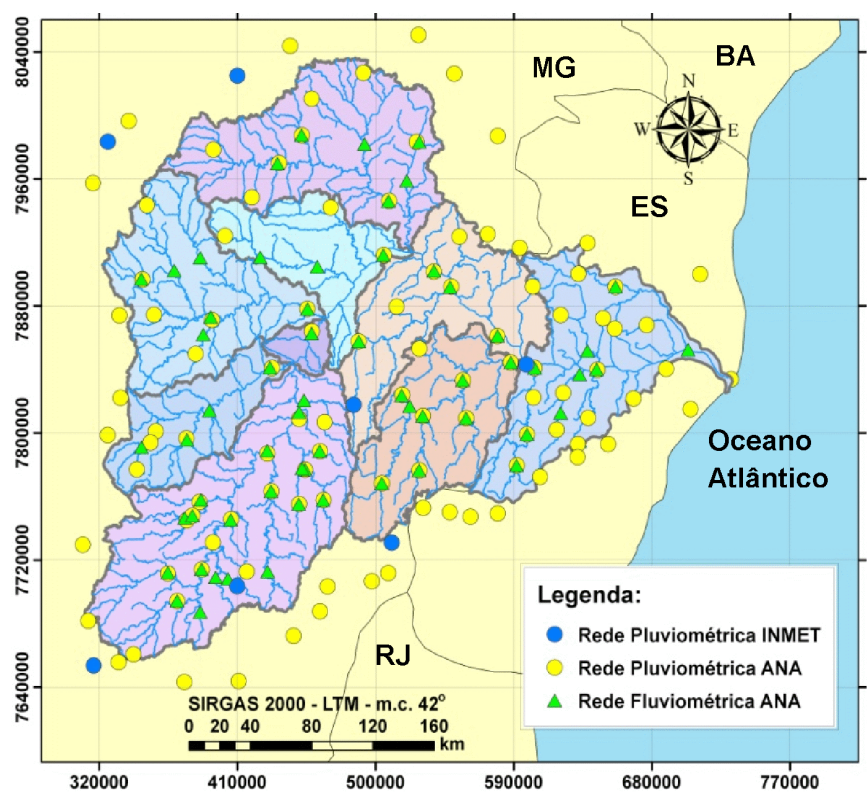

Figura 1. Distribuição espacial das estações de monitoramento consideradas no estudo

pelo Estado de Minas Gerais, abrangendo 84\% área da BHRD; já a variável $\mathrm{P}_{\mathrm{ss}}$ foi selecionada por se tratar de um evento com grande variabilidade no âmbito da bacia, segundo PIRH (2013).

Com vista à espacialização dos resultados utilizou-se o Sistema de Informações Geográficas ArcGIS 10.0 desenvolvido pelo Environmental Systems Research Institute - ESRI.

As análises estatísticas de tendência dos dados (tendência linear, quadrática e cúbica) foram realizadas com uso do aplicativo Statistica ${ }^{\circ} 7.0$ desenvolvido pela StatSoft; já para a obtenção dos semivariogramas teóricos e experimentais utilizou-se a ferramenta GEO-EAS (Englund \& Sparks, 1992).

\section{Proposta metodológica}

A primeira parte do fluxograma metodológico utilizado no projeto de redes de monitoramento hidrométrico é apresentada na Figura 2.

A proposta metodológica apresentada na Figura 2 se baseia na análise estatística descritiva preliminar dos dados hidrológicos e sua espacialização utilizando-se técnicas geoestatísticas. A análise estatística descritiva preliminar para as variáveis consideradas foi baseada nas seguintes avaliações:

- normalidade dos dados experimentais - aderência à distribuição Normal, segundo o teste de qui-quadrado $\left(\chi^{2}\right)$ a um nível de significância de 5\%;

- medidas de tendência central - média e mediana;

- análise de dispersão - análise de boxplot, desvio padrão e coeficiente de variação;

- superfícies de tendência - ajustes de superfícies de tendência de primeiro, segundo e terceiro graus aos dados de vazões específicas.

A normalidade dos dados indica a representatividade da média amostral e garante que, ao longo do espaço, seus valores médios são representativos. Segundo Landim (2003) não sendo satisfeita a condição de normalidade, as transformações das variáveis devem ser testadas, especialmente do tipo logarítmica. 


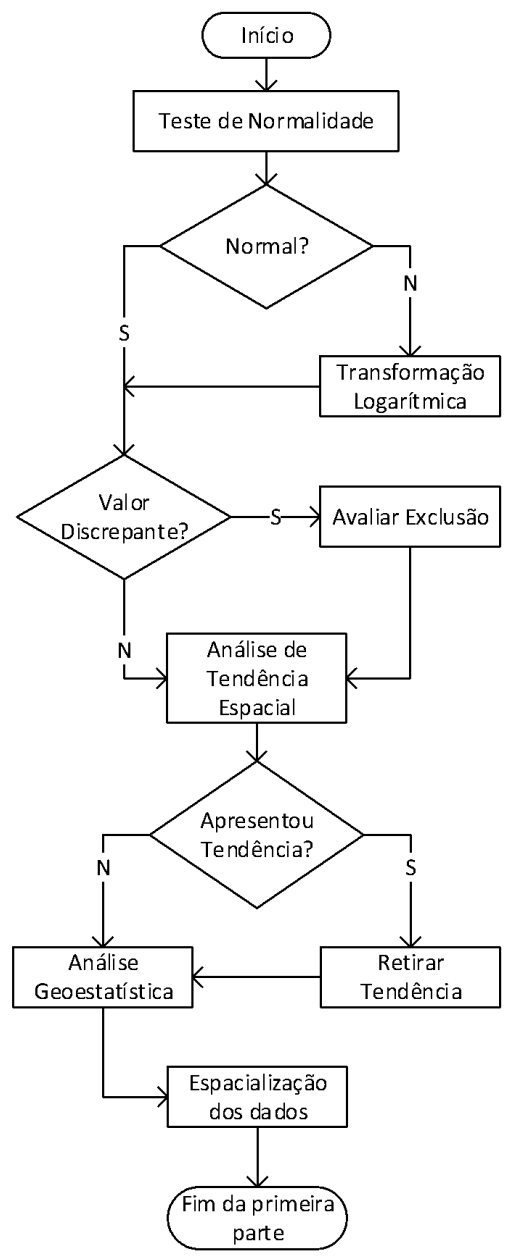

Figura 2. Fluxograma metodológico para projeto de redes de monitoramento hidrométrico - Parte I

O autor adverte que após qualquer transformação será preciso realizar, posteriormente, uma transformação inversa, trazendo os resultados para a distribuição original.

Foram realizadas as análises de medidas de tendência central e de dispersão com o intuito de se avaliar o grau de variabilidade espacial dos dados e a existência de possíveis valores discrepantes nas séries históricas verificando-se, também, os quartis da distribuição através da construção de box-plots.

A variabilidade espacial dos dados foi avaliada segundo proposta apresentada por Warrick \& Nielsen (1980) a partir do coeficiente de variação $(\mathrm{CV})$ os quais sugerem os limites $\mathrm{CV}<$ $12 \%, 12 \leq \mathrm{CV}<60 \%$ e $\mathrm{CV} \geq 60 \%$ para atributos de baixa, média e alta variabilidade, respectivamente.

Para Draper \& Smith (1981) a rejeição automática de valores discrepantes ou extremos não é procedimento correto e as regras propostas para sua rejeição devem incluir a reanálise sem essas observações que, dependendo das circunstâncias, podem conter informações essenciais e, portanto, não serem excluídas.

A análise de superfícies de tendências de $1^{\mathrm{a}}, 2^{\mathrm{a}}$ e $3^{\mathrm{a}}$ ordens, possibilitou a verificação da estacionariedade dos dados espacializados evitando estimativas espaciais tendenciosas e desvios padrão acentuados (Landim, 2003). A possibilidade de existência de tendência espacial nos dados avaliados foi verificada a partir do ajuste das superfícies de tendência aos dados avaliados pela metodologia dos mínimos quadrados.

Mencionadas tendências podem, em variáveis de vazão, ocorrer em razão de vários fatores, citando-se: precipitação, declividade, uso e ocupação do solo e disposição geográfica da rede de drenagem enquanto para variáveis de precipitação essas tendências são passíveis de ocorrer em virtude das diferenças de altitude, vegetação e temperatura.

O comportamento das variáveis regionalizadas na análise geoestatística foi avaliado a partir de duas ferramentas fundamentais: do semivariograma (análise do comportamento espacial das variáveis) e da krigagem (cálculo dos valores preditos com seus erros associados) conforme Mishra \& Coulibaly (2009).

Considerando que as variações espaciais de uma variável hidrológica regionalizada podem ser representadas pela realização $\mathrm{z}\left(\mathrm{x}_{\mathrm{i}}\right)$ de um conjunto $\mathrm{Z}(\mathrm{x})=\mathrm{z}_{1}(\mathrm{x}), \mathrm{z}_{2}(\mathrm{x}), \ldots, \mathrm{z}_{\mathrm{n}}(\mathrm{x})$, em que $x_{i}=\left(x_{i}, y_{i}\right)$ é o vetor localização da estação, o primeiro momento deste campo aleatório é o valor esperado para um ponto $\mathrm{E}[\mathrm{Z}(\mathrm{x})]$ e o segundo momento é definido como a covariância para qualquer par x e x', apresentado pela Eq. 1 (Journel \& Huijbregts, 1978):

$$
\mathrm{R}\left(\mathrm{x}, \mathrm{x}^{\prime}\right)=\mathrm{E}\left\{[\mathrm{z}(\mathrm{x})-\mathrm{E}[\mathrm{z}(\mathrm{x})]]\left[\mathrm{z}\left(\mathrm{x}^{\prime}\right)-\mathrm{E}\left[\mathrm{z}\left(\mathrm{x}^{\prime}\right)\right]\right]\right\}
$$

A função semivariograma é definida por dois valores separados por uma distância h denominada "lag". Assim, a Eq. 2 pode ser calculada pelo semivariograma experimental clássico assumindo-se estacionariedade:

$$
\begin{gathered}
\gamma(\mathrm{x}, \mathrm{h})=\mathrm{E}\left\{[\mathrm{z}(\mathrm{x})-\mathrm{z}(\mathrm{x}+\mathrm{h})]^{2}\right\} \\
\hat{\gamma}(\mathrm{h})=\frac{1}{2 \mathrm{~N}} \sum_{\mathrm{i}=1}^{\mathrm{N}}\left[\mathrm{z}\left(x_{i}\right)-\mathrm{z}\left(x_{i}+\mathrm{h}\right)\right]^{2}
\end{gathered}
$$

em que:

$\mathrm{N}$ - número total de pares

$\mathrm{z}\left(\mathrm{x}_{\mathrm{i}}\right)$ - variável de interesse

h - distância de separação entre duas variáveis

$\gamma(\mathrm{h})$ - semivariograma espacial clássico

Um mínimo de 30 pares de pontos por classe de distância foi utilizado para se obter uma estimativa adequada da semivariância experimental (Journel \& Huijbregts, 1978).

Com base nas semivariâncias experimentais calculadas escolheu-se, dentre os modelos de semivariograma exponencial, gaussiano e esférico, aquele que melhor representou a variabilidade espacial em análise.

A krigagem baseou-se no número de dados, na qualidade dos dados em cada ponto, na posição da coleta dos dados, na continuidade espacial e na distância entre esses pontos amostrais (Journel \& Huijbregts, 1978).

$\mathrm{Na}$ krigagem os valores estimados de $\mathrm{z}\left(\mathrm{x}_{0}\right)$ identificados por uma localização específica $\mathrm{x}_{0}$ na qual não existem valores, foram dados pela Eq. 4: 


$$
\hat{z}\left(x_{0}\right)=\sum_{i=1}^{N} \lambda_{i} z\left(x_{i}\right)
$$

em que:

$\lambda_{i} \quad$ - pesos do estimador que minimizam a variância (pesos da Krigagem ordinária)

Supondo que $\mathrm{z}\left(\mathrm{x}_{\mathrm{i}}\right)$ satisfaz à hipótese $\mathrm{E}[\mathrm{z}(\mathrm{x})-\mathrm{z}(\mathrm{x}+\mathrm{h})]=0 \mathrm{e}$ possui semivariograma dado pela Eq. 2 , um sistema de equações lineares de krigagem simples combinando informações de vizinhança, foi assim definido:

$$
\begin{gathered}
\sum_{i=1}^{N} \lambda_{i} \gamma\left(x_{i}-x_{j}\right)=\gamma\left(x_{i}-x_{0}\right) \\
\sum_{i=1}^{N} \lambda_{i}=1
\end{gathered}
$$

Segundo Landim (2003) a krigagem fornece, em média, estimativas não tendenciosas (a diferença entre valores estimados e verdadeiros para o mesmo ponto, em média, deve ser nula) e com variância mínima. A krigagem, portanto, garante a melhor estimativa espacial possível caso seja identificada a dependência espacial das variáveis analisadas a partir dos semivariogramas teóricos obtidos.

Para a validação do semivariograma teórico ajustado utilizou-se a técnica de Jack-knifing ou validação cruzada (Vauclin et al., 1983). Nesta técnica cada um dos valores medidos é interpolado pelo método da krigagem eliminandose sucessivamente os valores medidos, substituindo-os pelas estimativas e se calculando, a seguir, a distribuição dos erros padronizados que deve apresentar média zero e desvio-padrão unitário (Montenegro \& Montenegro, 2006).

A aplicação de todas as etapas metodológicas apresentadas possibilita a espacialização não tendenciosa de dados hidrológicos permitindo estimar dados em regiões não monitoradas e com os menores erros possíveis.

\section{Resultados e Discuss ão}

A análise estatística dos dados para as 61 estações de monitoramento fluviométrico e para as 112 estações de monitoramento pluviométrico é apresentada na Tabela 1.

Tabela 1. Análise estatística das variáveis hidrológicas em estudo

\begin{tabular}{lccc}
\hline \multicolumn{1}{c}{ Estatísticas } & $\begin{array}{c}\mathbf{q}_{\mathbf{7}, 10} \\
\left(\mathbf{L ~ s}^{-1} \mathbf{k m}^{-2} \mathbf{)}\right.\end{array}$ & $\begin{array}{c}\mathbf{P}_{\mathbf{s s}} \\
(\mathbf{m m})\end{array}$ & $\log _{\mathbf{s s}}$ \\
NN$^{0}$ de observações & 61 & 112 & 112 \\
Média & 3,36 & 198,13 & 2,288 \\
Mediana & 3,38 & 190,63 & 2,280 \\
Mínimo & 0,33 & 129,25 & 2,111 \\
Máximo & 9,46 & 461,55 & 2,664 \\
Desvio-padrão & 1,84 & 44,04 & 0,087 \\
Coeficiente de variação (\%) & 54,64 & 22,23 & 3,80 \\
\hline
\end{tabular}

$q_{710}$ - Vazão específica mínima média de sete dias consecutivos e período de retorno de 10 anos, $\mathrm{P}_{\mathrm{ss}}$ - Precipitação total do semestre mais seco e $\log \mathrm{P}_{\mathrm{ss}}$ - Logaritmo da precipitação total do semestre mais seco
Com base na proposta apresentada por Warrick \& Nielsen (1980) para classificação da variabilidade dos dados a partir do coeficiente de variação $(\mathrm{CV})$ a vazão específica mínima $\mathrm{q}_{7,10}$ $(\mathrm{CV}=54,64 \%)$ e a precipitação $\mathrm{P}_{\mathrm{ss}}(\mathrm{CV}=22,23 \%)$ apresentaram variabilidade espacial média para a BHRD.

Utilizando-se o teste de qui-quadrado, a hipótese de normalidade pôde ser aceita para a variável $\mathrm{q}_{7,10}$ a um nível de significância de $5 \%$. Já para a variável $\mathrm{P}_{\mathrm{ss}}$ esta hipótese não foi aceita. A análise estatística da variável transformada $\log \mathrm{P}_{\mathrm{ss}}$ também é apresentada na Tabela 1.

Após a transformação logarítmica pôde-se considerar a variabilidade espacial dos dados de $\mathrm{P}_{\mathrm{ss}}$ como baixa $(\mathrm{CV}=3,80 \%)$ e a hipótese de normalidade, segundo o teste de aderência de qui-quadrado a um nível de significância de 0,05 , pôde ser aceita.

Garantindo a normalidade das variáveis, avaliou-se a existência de valores discrepantes nas séries consideradas a partir da análise de box-plot e só então se observou que as variáveis $\mathrm{q}_{7,10}$ e $\log \mathrm{P}_{\text {ss }}$ apresentaram valores discrepantes que se referem às estações fluviométricas 56337000 e $56335001 \mathrm{e}$ pluviométricas 01940005 e 01939002 , respectivamente. Após análise proposta por Draper \& Smith (1981) concluiu-se que os dados dessas estações eram consistentes não sendo, portanto, descartados para o restante do estudo.

Antes de realizar a análise geoestatística das redes fluviométrica e pluviométrica verificou-se a possibilidade da existência de tendência espacial nos dados avaliados. Foram testados os ajustes das superfícies de tendência de $1^{\circ}$, $2^{\circ}$ e $3^{\circ}$ graus, tendo sido descartada a possibilidade de ajuste de tendência linear, quadrática e cúbica para as variáveis hidrológicas avaliadas, por terem apresentado coeficientes de determinação $\left(\mathrm{r}^{2}\right)$ menores que 0,55 (Santos, 2007).

A não existência de tendência nos dados originais de vazão e precipitação permitiu a análise geoestatística não tendenciosa das variáveis aumentando a confiabilidade dos dados a serem espacializados.

\section{Análise geoestatística}

Com base nas 61 estações de monitoramento fluviométrico e nas 112 estações de monitoramento pluviométrico, estimaramse as semivariâncias experimentais da $\mathrm{q}_{7,10}$ e $\mathrm{P}_{\text {ss }}$, utilizando-se o estimador clássico (Eq. 3).

Para a variável $\mathrm{q}_{7,10}$ foram utilizados o incremento (lag) de 13 $\mathrm{km}$, a distância de separação mínima de $10 \mathrm{~km}$ e a distância de separação máxima de $100 \mathrm{~km}$. A variável $\mathrm{P}_{\mathrm{ss}}$ foi preliminarmente transformada logaritmicamente para garantir a normalidade dos dados de chuva. Para a variável $\log \mathrm{P}_{\text {ss }}$ se utilizaram o incremento de $21 \mathrm{~km}$ e a distância de separação máxima de $190 \mathrm{~km}$.

Após a obtenção dos semivariogramas experimentais procurou-se ajustar os modelos teóricos através dos quais fosse mais bem caracterizado o comportamento espacial das variáveis estudadas. Assumiu-se a isotropia do domínio no tocante à estrutura de dependência espacial. As variáveis, os parâmetros dos modelos e os modelos ajustados podem ser visualizados na Tabela 2. 
Tabela 2. Parâmetros dos modelos de semivariogramas teóricos ajustados aos dados experimentais de $q_{7,10}$ e $\mathrm{P}_{\mathrm{ss}}$

\begin{tabular}{lcc}
\hline \multicolumn{1}{c}{ Parâmetros } & $\mathbf{q}_{7,10}$ & $\log \mathbf{P}_{\mathrm{ss}}$ \\
Efeito Pepita $\left(\mathrm{C}_{0}\right)$ & 0,400 & 0,0027 \\
Patamar $\left(\mathrm{C}_{0}+\mathrm{C}_{1}\right)$ & 2,300 & 0,0038 \\
Alcance $(\mathrm{m})$ & 60.000 & 120.000 \\
$\mathrm{C}_{0} /\left(\mathrm{C}_{0}+\mathrm{C}_{1}\right)^{*}$ & 17,39 & 41,54 \\
Modelo & Esférico & Esférico \\
\hline
\end{tabular}

$\mathrm{q}_{710}$ - Vazão específica mínima média de sete dias consecutivos e período de retorno de 10 anos e logPss - Logaritmo da precipitação total média do semestre mais seco; ${ }^{*} C_{d} /\left(C_{0}+\right.$ $C_{1}$ ) - Grau de dependência espacial de Cambardella et al. (1994) em (\%)

Analisando a Tabela 2, observa-se que o modelo esférico se ajustou adequadamente às semivariâncias experimentais das variáveis estudadas apresentando-se melhor que os demais modelos testados. Verificam-se, ainda, nos ajustes dos semivariogramas teóricos obtidos, alcances de $60 \mathrm{~km}$ para $\mathrm{q}_{7,10}$ e $120 \mathrm{~km}$ para $\log \mathrm{P}_{\text {ss }}$. Segundo Landim (2003) os alcances fornecem o tamanho da zona de influência em torno de uma amostra.

Era de se esperar que a precipitação tivesse alcance maior que a vazão devido ao processo físico e hidrológico inerente às variáveis. A precipitação, quando não sujeita à influência orográfica, pode englobar grandes áreas e abranger uma ou mais bacias hidrográficas adjacentes; já a vazão é altamente influenciadada pela área de drenagem na qual está inserida, espacialmente definida pelos divisores de água da bacia.

Segundo a relação entre efeito pepita e patamar proposta por Cambardella et al. (1994) a variável $\mathrm{q}_{7,10}$ apresentou forte dependência espacial (menor que 25\%) com 17,39\% enquanto a variável $\log \mathrm{P}_{\mathrm{ss}}$ apresentou, segundo os autores, dependência espacial moderada (entre 25 e $75 \%$ ) com grau de $41,54 \%$.

A verificação do modelo teórico ajustado (esférico) foi feita a partir do método de validação cruzada (Vauclin et al., 1983). Na Tabela 3 são apresentados as médias e os desvios padrão dos resíduos produzidos pela validação cruzada para as variáveis estudadas.

Analisando os resultados apresentados na Tabela 3 observa-se que as médias e os desvios padrão dos resíduos foram considerados suficientemente próximos de zero e um, respectivamente, validando os modelos teóricos ajustados para as variáveis consideradas.

Aplicando a técnica de interpolação por Krigagem a partir dos semivariogramas teóricos ajustados, se obtiveram os mapas espacializados (Figuras 3 e 4) para $\mathrm{q}_{7,10}$ e $\mathrm{P}_{\text {ss }}$, respectivamente. A transformação logarítmica inversa foi aplicada para apresentação dos dados espacializados da variável $\mathrm{P}_{\mathrm{ss}}$.

Analisando a Figura 3 verifica-se que, de forma geral, os valores da variável $\mathrm{q}_{7,10}$ na BHRD, diminuem de oeste para

Tabela 3. Estatística dos resíduos produzidos pela validação cruzada para $\mathrm{q}_{7,10}$ e $\log \mathrm{P}_{\mathrm{ss}}$

\begin{tabular}{ccc}
\hline Resíduos & $\mathbf{q}_{7,10}$ & $\log \mathbf{P}_{\mathrm{ss}}$ \\
Média & 0,021 & 0,014 \\
Desvio padrão & 1,068 & 1,090 \\
\hline
\end{tabular}

Legenda: $q_{710}$-Vazão específica mínima média de sete dias consecutivos e período de retorno de 10 anos e $\log \mathrm{P}_{\mathrm{ss}}$ - Logaritmo da precipitação total média do semestre mais seco

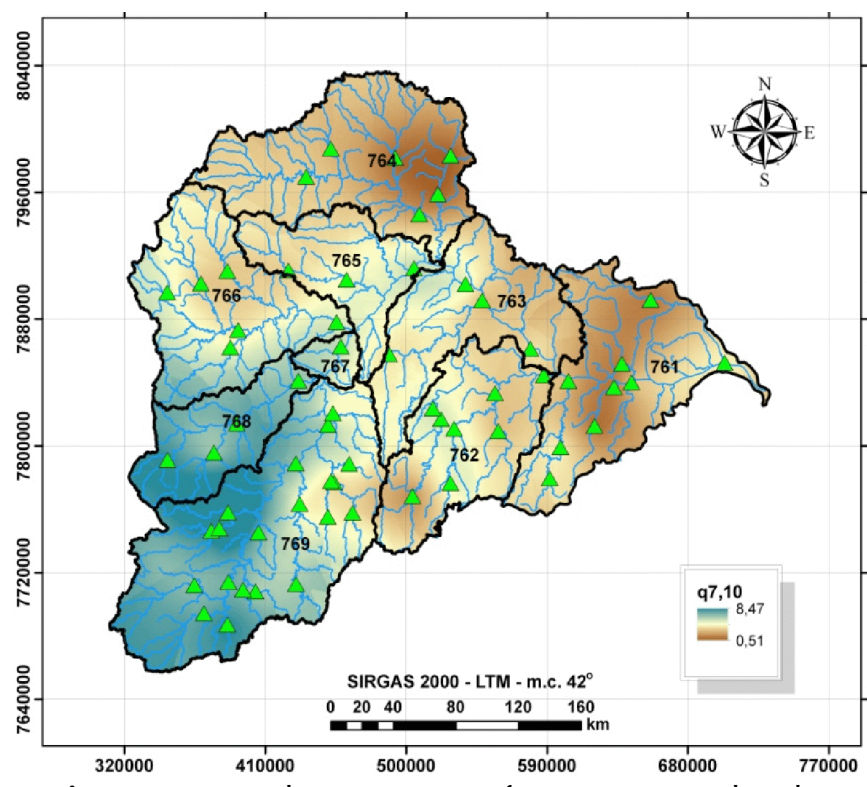

Figura 3. Mapa de vazão específica $q_{7,10}$ espacializada, em $\mathrm{L} \mathrm{s}^{-1} \mathrm{~km}^{-2}$

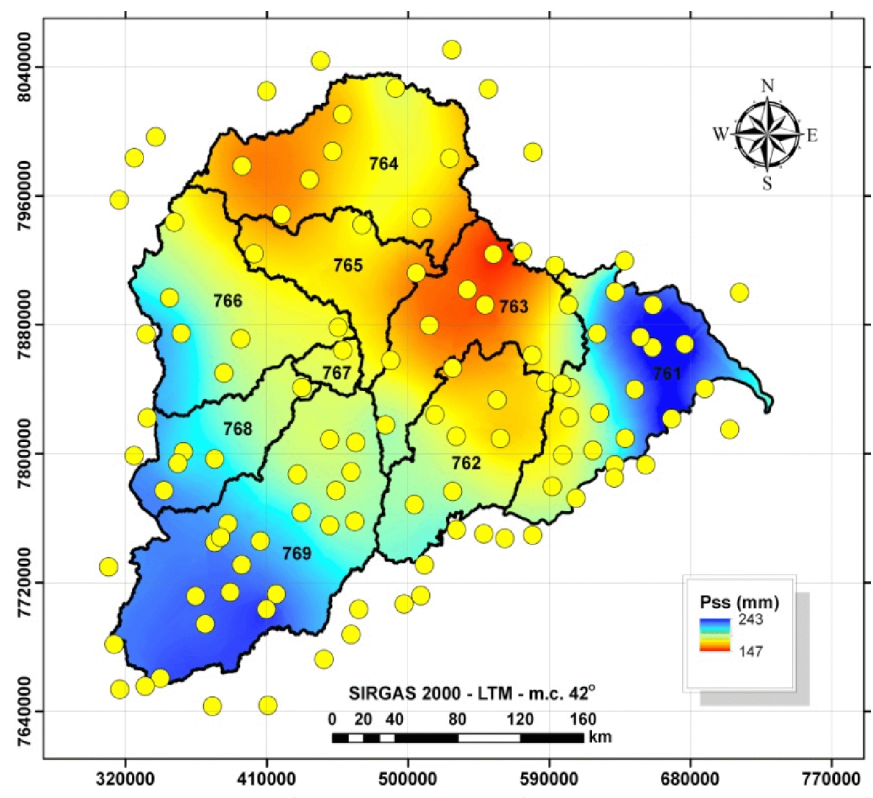

Figura 4. Mapa de precipitação do semestre mais seco $\left(\mathrm{P}_{\mathrm{ss}}\right)$ espacializada, em $\mathrm{mm}$

leste, das regiões mais declivosas e com índices de precipitação anual maiores para as regiões menos declivosas e com índices de precipitação anual menores. $\mathrm{Na} B H R D$, a estimativa da vazão específica $\mathrm{q}_{7,10}$, variou de 0,51 a $8,47, \mathrm{em} \mathrm{L} \mathrm{s}^{-1} \mathrm{~km}^{-2}$.

Em relação à Figura 4 pode-se observar que a variável $\mathrm{P}_{\text {ss }}$ apresentou maiores valores na região de cabeceira do rio Piranga e do baixo rio Doce (ottocódigos 769 e 761) e precipitações mínimas maiores na região central da BHRD (ottocódigos 763, 764 e 765). A estimativa de $\mathrm{P}_{\mathrm{ss}}$ variou de 147a $243 \mathrm{~mm}$.

A primeira parte da metodologia proposta para o projeto de redes hidrométricas com vistas à espacialização não tendenciosa dos dados hidrológicos, adequou-se aos dados de vazão $\mathrm{q}_{7,10} \mathrm{e}$ precipitação $\mathrm{P}_{\text {ss }}$ da $\mathrm{BHRD}$. 


\section{Conclusões}

1. A aplicação da metodologia proposta permitiu a espacialização não tendenciosa das variáveis vazão específica mínima média de sete dias consecutivos e período de retorno de 10 anos $\left(\mathrm{q}_{7,10)}\right.$ e precipitação total do semestre mais seco $\left(\mathrm{P}_{\mathrm{ss}}\right)$ na bacia do rio Doce.

2. A metodologia permitiu a estimativa de dados em regiões não monitoradas com os menores erros possíveis.

\section{Agradecimentos}

Os autores agradecem à Universidade Federal de Viçosa (UFV), à Fundação de Amparo à Pesquisa do Estado de Minas Gerais (FAPEMIG), à Coordenação de Aperfeiçoamento de Pessoal de Nível Superior (CAPES) e ao Conselho Nacional de Desenvolvimento Científico e Tecnológico (CNPq) pelo financiamento deste trabalho.

\section{Literatura Citada}

Anctil, F.; Lauzon, N.; Andréassuan V.; Oudin, L.; Perrin, C. Improvement of rainfall-runoff forecasts through mean areal rainfall optimization. Journal of Hydrology, v.328, p.717-725, 2006.

Bardossy, A.; Das, T. Influence of rainfall observation network on model calibration and application. Hydrology and Earth System Sciences, v.12, p.77-89, 2008.

Cambardella, C.A.; Moorman, T. B.; Novak, J. M.; Parkin, T. B.; Karlen, D. L.; Turco, R. F.; Konopca, A. E. Field-scale variability of soil properties in central Iowa soils. Soil Science Society America Journal, v.58, p.1240-1248, 1994.

Coulibaly, P. Spatial and temporal variability of Canadian seasonal precipitation (1900-2000). Advances in Water Resources, v.29, p.1846-1865, 2006.

Coulibaly, P., Evora, D.N. Comparison of neural network methods for infilling missing daily weather records. Journal of Hydrology, v.341, p.27-41, 2007.

Draper, N. R.; Smith, H. Applied regression analysis. 2.ed. New York: John Wiley, 1981. 709p.

Elesbon, A. A. A.; Guedes, H. A. S.; Silva, D. D.; Castro, I. O. Uso de dados SRTM e plataforma SIG na caracterização morfométrica da bacia hidrográfica do braço norte do rio São Mateus - Brasil. Revista Escola de Minas, v.64, p.281288, 2011.

Englund, E.; Sparks, A. Geo-EAS: Geostatistical Environmental Assessment Software. Las Vegas: International Ground Water Modeling Center. 1992. CD-Rom.

Gerstner, E. M.; Heinemann, G. Real-time areal precipitation determination from radar by means of statistical objective analysis. Journal of Hydrology, v.352, p.296-308, 2008.
Guedes, H. A. S.; Silva, D. D.; Elesbon, A. A. A.; Ribeiro, C. B. M.; Matos, A. T.; Soares, J. H. P. Aplicação da análise estatística multivariada no estudo da qualidade da água do rio Pomba, MG. Revista Brasileira de Engenharia Agrícola e Ambiental. v.16, p.558-563, 2012.

Journel, A. G.; Huijbregts, C. J. Mining geostatistics. London: Academic Press, 1978. 600p.

Landim, P. M. B. Análise geoestatística de dados geológicos. São Paulo: UNESP, 2.ed., 2003. 253p.

Ludwig, R.; Schneider, P. Validation of digital elevation models from SRTM X- SAR for applications in hydrologic modeling. Journal of Photogrammetry \& Remote Sensing, v.60, p.339-358, 2006.

Machado, R. D.; Rocha, R. P. Previsões climáticas sazonais sobre o Brasil: avaliação do RegCM3 aninhado no modelo global CPTEC/COLA. Revista Brasileira de Meteorologia, v.26, p.121-136, 2011.

Mishra, A. K.; Coulibaly, P. Hydrometric network evaluation for Canadian watersheds. Journal of Hydrology, v.380, p.420-437, 2009.

Mishra, A. K., Ozger, M., Singh, V. P. An entropy based investigation into the variability of precipitation. Journal of Hydrology, v.370, p.139-154, 2009.

Montenegro, A. A. A.; Montenegro, S. M. G. L. Variabilidade espacial de classes de textura, salinidade e condutividade hidráulica de solos em planície aluvial. Revista Brasileira de Engenharia Agrícola e Ambiental, v.10, p.30-37, 2006.

Oliveira, P. T. S. de; Alves Sobrinho, T.; Steffen, J. L.; Rodrigues, D. B. B. Caracterização morfométrica de bacias hidrográficas através de dados SRTM. Revista Brasileira de Engenharia Agrícola e Ambiental, v.14, p.819-825, 2010.

PIRH - Plano Integrado de Recursos Hídricos da Bacia do Rio Doce. <http://www.pirhdoce.com.br/>. 10 Mar. 2013.

Rogalus, M. J.; Ogden, F. L. Comparison of GCIP and stage III radar-rainfall estimate over the Mississipi River Basin for 1997. Journal of Hydrology, v.341, p.177-185, 2007.

Santos, C. Estatística descritiva: Manual de autoaprendizagem. Lisboa: Silabo, 1.ed., 2007. 264p.

Valeriano, M. M.; Kuplich, T. M.; Storino, M.; Amaral, B. D.; Mendes Júnior, J. N.; Lima, D. J. Modeling small watersheds in Brazilian Amazônia with SRTM-90m data. Computer \& Geosciences, v.32, p.1169-1181, 2006.

Vauclin, M.; Vieira, S. R.; Vachaud, G.; Nielsen, D. R. The use of cokriging with limited field soil observations. Soil Science Society of America Journal, v. 47, p.175-184, 1983.

Warrick, A. W.; Nielsen, D. R. Spatial variability of soil physical properties in the field. Applications of soil physics. In: Hillel, D., ed. Application of soil physics. New York: Academic Press, 1980. 385p.

Westcott, N. E.; Knapp, H. V.; Hilmerg, S. D. Comparison of gage and multi-sensor precipitation estimates over a range of spatial and temporal scales in the Midwestern United States. Journal of Hydrology, v.351, p.1-12, 2008. 